\title{
UCRL-PROC-209970
}

LAW RENCE LIVERMORE N A T IO N A L LABORATORY

\section{Oxidation resistance of Ru-capped EUV multilayers}

S. Bajt, Z. Dai, E. J. Nelson, M. A. Wall, J. Alameda, N. Nguyen, S. Baker, J. C. Robinson, J. S. Taylor, M. Clift, A. Aquila, E. M. Gullikson, N. V. G. Edwards

February 25, 2005

SPIE 30th International Symposium

San Jose, CA, United States

February 27, 2005 through March 4, 2005 
This document was prepared as an account of work sponsored by an agency of the United States Government. Neither the United States Government nor the University of California nor any of their employees, makes any warranty, express or implied, or assumes any legal liability or responsibility for the accuracy, completeness, or usefulness of any information, apparatus, product, or process disclosed, or represents that its use would not infringe privately owned rights. Reference herein to any specific commercial product, process, or service by trade name, trademark, manufacturer, or otherwise, does not necessarily constitute or imply its endorsement, recommendation, or favoring by the United States Government or the University of California. The views and opinions of authors expressed herein do not necessarily state or reflect those of the United States Government or the University of California, and shall not be used for advertising or product endorsement purposes. 


\title{
Oxidation resistance of Ru-capped EUV multilayers
}

\author{
Saša Bajt ${ }^{* a}$, Zurong Dai ${ }^{a}$, Erik J. Nelson ${ }^{a}$, Mark A. Wall ${ }^{a}$, Jennifer Alameda ${ }^{a}$, Nhan Nguyen ${ }^{a}$, \\ Sherry Baker ${ }^{\mathrm{a}}$, Jeffrey C. Robinson ${ }^{\mathrm{a}}$, John S. Taylor ${ }^{\mathrm{a}}$, Miles Clift $\mathrm{b}^{\mathrm{b}}$, Andy Aquila ${ }^{\mathrm{c}}$, \\ Eric M. Gullikson ${ }^{\mathrm{c}}$, and N. V. Ginger Edwards ${ }^{\mathrm{d}}$ \\ a'Lawrence Livermore National Laboratory, 7000 East Avenue, Livermore, CA, USA 94550; \\ ${ }^{\mathrm{b}}$ Sandia National Laboratories, 7011 East Avenue, Livermore, CA, USA 94550; \\ ${ }^{\mathrm{C}}$ Lawrence Berkeley National Laboratory, 1 Cyclotron Road, Berkeley, CA, USA 94720; \\ ${ }^{\mathrm{d}}$ SEMATECH, Austin, TX, USA 78741-6499
}

\begin{abstract}
Differently prepared Ru-capping layers, deposited on Mo/Si EUV multilayers, have been characterized using a suite of metrologies to establish their baseline structural, optical, and surface properties in as-deposited state. Same capping layer structures were tested for their thermal stability and oxidation resistance. Post-mortem characterization identified changes due to accelerated tests. The best performing Ru-capping layer structure was studied in detail with transmission electron microscopy to identify the grain microstructure and texture. This information is essential for modeling and performance optimization of EUVL multilayers.
\end{abstract}

Keywords: EUVL, multilayer, capping layer, $\mathrm{Mo} / \mathrm{Si}$, Ru, microstructure, oxidation, thermal stability

\section{INTRODUCTION}

The lifetime of projection and condenser optics remains a critical issue for commercializing EUVL (extreme ultraviolet lithography) technology. At a recent EUVL symposium (Miyazaki, Japan, Nov. 2004), the lifetime of the projection and illuminator optics was again voted as one of the most critical issues for commercial introduction of EUV lithography. Since the illuminator and the projection optics operate in different environments, the solutions to extend their lifetime are likely to be different. In this paper we are discussing only protective layers appropriate for projection optics and the properties of one potential candidate, $\mathrm{Ru}$.

Water and hydrocarbons are two major contamination sources that reduce the lifetime of projection optics when exposed to the EUV light. Different solutions to mitigate surface contamination from outgassing have been explored [13]. Oxidation due to the presence of water vapor in the EUV lithography tools is considered a more serious problem because the process is considered to be irreversible. The interaction between EUV photons and multilayer material creates secondary electrons in the top layers of the multilayer. The secondary electrons that reach the surface of the multilayer and have sufficient energy to break the bonds in water molecules will create free radicals. These radicals can bond to the capping layer atoms and form oxides on the surface or diffuse into or through the top layer and cause oxidation. Oxygen strongly absorbs EUV light and even a small increase in oxide layer thickness $(<0.3 \mathrm{~nm})$ on the reflective optics leads to a measurable reflectance loss. One way to mitigate oxidation is to introduce gas-phase ethanol in the system to form a self-limiting protective surface carbon layer [4-5] or use the ethanol to simultaneously mitigate the growth speed of carbon on a proprietary capping layer [3]. Alternatively, one can use oxidation protective capping layers. Extended lifetimes have been reported for ruthenium [6-7] and carbon capping layers [8-9]. However, a high reflectivity solution with no less than $1 \%$ reflectivity change over 5 years of commercial tool operation has yet to be demonstrated.

To understand what makes some materials better oxidation capping layers than the others one needs to study their microstructure. It is well known that the properties of thin films differ from the properties of bulk materials and the

\footnotetext{
*bajt@llnl.gov; phone 1925424 3768; fax 19254231488
} 
microstructure needs to be studied on realistic thin $(2-3 \mathrm{~nm})$ capping layers, which are deposited on the top of a multilayer structure. In this paper we are attempting to identify the microstructure and texture of the best performing Ru capping layers. The study of detailed oxidation mechanisms is beyond the scope of this work.

\section{METHODOLOGY}

All samples are based on a standard 50 bilayer Mo/Si multilayer deposited on 100-mm diameter Si (100) superpolished wafers. These substrates have typical surface high spatial frequency (HSF) roughness of $0.1 \mathrm{~nm}$ or better. The multilayer bilayer thickness (the combined Mo and Si layer thickness) was kept constant for all samples at about 6.94 $\mathrm{nm}$. This period thickness is required for operation of these multilayers at $13.4 \mathrm{~nm}$ at near normal incidence. Molybdenum thickness divided by the period thickness, also called $\Gamma$, was kept constant at 0.4 . All depositions were performed in a dc-magnetron sputtering system (Mag3) using three or four sputtering sources, depending on the capping layer structure.

Five different preparation techniques were used to deposit Ru capping layers on Mo/Si-based EUVL multilayers, also called Multilayer 1. Modifications include power change (Preparation 1), sputtering gas change (Preparation 4, 5 and 6) and material variation (Preparation 7). Mo/Si basic multilayer structure was made the same in all cases using ultra-pure Ar sputtering gas. The Ru capping layer in Preparation 1 was prepared using Ar sputtering gas. Ru capping layers for Preparation 4 and Preparation 5 samples were deposited in Ar: $\mathrm{N}_{2}$ gas mixtures with different $A r: \mathrm{N}_{2}$ ratios. Preparation 6 capping layer was deposited with pure Ar gas but exposed to pure $\mathrm{N}_{2}$ environment for about 15 minutes before being removed from the deposition tool. The Ru capping layer in Preparation 7 was doped with carbon.

These multilayer coatings have been characterized in the as-deposited state using a suite of metrologies to establish their baseline information. Multilayers, and in particular, their capping layer structures were then tested for thermal stability and oxidation resistance. We refer to these tests as screening tests. The main objective was to identify the best candidate for further scaling, parametric and accelerated studies.

Multilayer period thickness and detailed multilayer structure (interface roughness, surface roughness, individual layer thicknesses and densities) were determined using $\mathrm{x}$-ray diffractometry with a $\mathrm{Cu} \mathrm{K}_{\alpha}$ source $(\lambda=0.15 \mathrm{~nm})$ and fitting these data with model calculations. Furthermore, surface high spatial frequency rms roughness was obtained with AFM using $2 \times 2 \mathrm{~m}^{2}$ scans. A reflectometer, at beamline 6.3.2. (Advanced Light Source, LBNL) was used to measure reflectivity as a function of wavelength and to map the samples for their reflectivity variations at a fixed wavelength. The best performing capping layer was studied with Philips CM300 FEG transmission electron microscope (TEM) at LLNL operating at $300 \mathrm{kV}$. Thermal testing was performed in vacuum at $100^{\circ} \mathrm{C}$ for 1 week and at $200^{\circ} \mathrm{C}$ for 30 minutes. The oxidation resistance test was performed using electron beam exposures in a water vapor environment. The exposures were performed in Auger microprobes at Sandia National Laboratories (SNL) and Lawrence Livermore National Laboratory (LLNL) using a focused electron beam with $1 \mathrm{keV}$ electrons that was rastered over about $0.6 \mathrm{x} 0.6$ $\mathrm{mm}^{2}$. The current density within this area was kept at $\sim 5 \mu \mathrm{A} / \mathrm{mm}^{2}$. The exposures were done at $5 \times 10^{-7}$ Torr of water vapor pressure with no other detectable contaminant in the system, and all the exposures were 40 hours long. The electron beam exposed samples were analyzed using the same techniques as described above. In addition X-ray Photoelectron Spectroscopy (XPS) was used to determine the surface chemistry inside and outside the exposed area. Reflectance maps at a fixed wavelength were collected on all exposed samples with the beam spot size of about $300 \mathrm{x}$ $50 \mu^{2}$, which was sufficient to resolve the exposed area. Auger electron spectroscopy was used to identify the elemental composition and to perform depth profiling. An ion beam sputter source was used to remove less than one monolayer of material from the surface at a time during continued monitoring of the composition and chemistry of the multilayer.

\section{DATA AND RESULTS}

The effect of different capping layers is clearly demonstrated in their initial reflectivity, as shown in Figure 1. Among the five samples, Preparation 7 has the highest pre-exposure reflectivity (66.7\%) and Preparations 4 and 5 have the lowest pre-exposure reflectivities ( 64\%). We noticed two factors that affect the reflectivity results. The initial reflectivity is sensitive to the chamber base pressure. Since the main contaminant remaining in the chamber is usually 


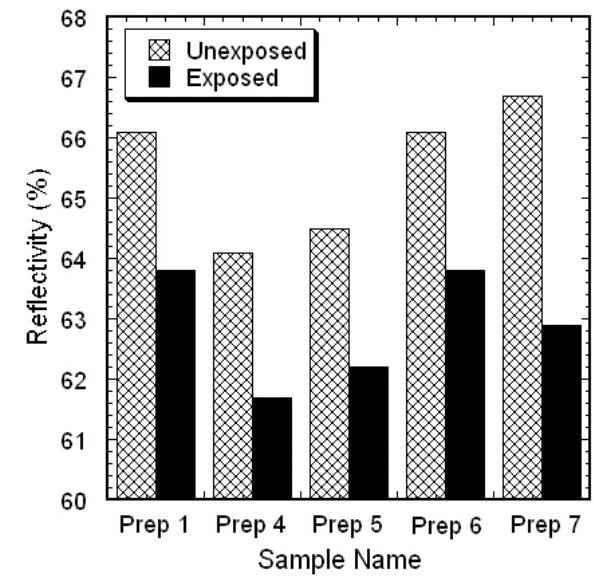

Figure 1: Initial absolute reflectivity (unexposed) ranges between $64.1 \%$ (Preparation 4) to 66.7\% (Preparation 7) due to different preparation techniques. In the electron beam exposed areas (exposed) the reflectivity is always lower. The largest reflectivity drop was observed for Preparation 7 sample.
$\mathrm{H}_{2} \mathrm{O}$, it is of no surprise that a slightly lower base pressure leads to slightly higher reflectivity. For example, the samples with the chamber base pressure in $\sim 2 \times 10^{-7}$ Torr had as much as $0.5 \%$ lower reflectivity than the samples with the same design made with the chamber base pressure at $\sim 8 \times 10^{-8}$ Torr. We also observed that the reflectivity of Ru-capped multilayer mirrors changes with time. Measuring the same multilayer mirror over a period of one year showed that the reflectivity drops as a function of time (Figure 2). Ru-capped multilayer (Preparation 1) shows the highest reflectance drop in the first few weeks after the multilayer deposition, similar to what was observed on Sicapped multilayers[10]. A relatively small fraction of the reflectivity drop $(>0.5 \%)$ is probably due to atomic rearrangement on the interfaces within the multilayer that also leads to a small $(0.02 \mathrm{~nm})$ wavelength shift. The second contribution to reflectivity loss is likely due to surface contamination and oxidation. As shown in Figure 1, the reflectance dropped by more than $1 \%$ in the first 2 months after the deposition but remained relatively stable after that. The data set covers a period of one year. However, apart from the small initial wavelength shift there were no subsequent changes in the peak wavelength and peak width. The multilayers were aged in the air, kept in a plastic container and were not cleaned between the measurements. All unexposed reflectivity values in Figure 2 refer to multilayers aged in the air for at least 2 months. These multilayers presumably reached a steady-state reflectivity before they were tested for oxidation resistance. It is interesting to note that the reflectivity of Preparation 7 was initially as high as $68.3 \%$, when measured immediately after deposition, thus comparable to or even exceeding the reflectivity of Si-capped multilayer of the same design.

Table 1: High spatial frequency (HSF) roughness measured with AFM on as-deposited and thermally annealed samples.

\begin{tabular}{|cccc|}
\hline Preparation & $\begin{array}{c}\text { As-deposited } \\
(\mathrm{nm} \mathrm{rms})\end{array}$ & $\begin{array}{c}100^{\circ} \mathrm{C} \text { for } 1 \text { week } \\
(\mathrm{nm} \mathrm{rms})\end{array}$ & $\begin{array}{c}200^{\circ} \mathrm{C} \text { for } 30 \text { min } \\
(\mathrm{nm} \text { rms })\end{array}$ \\
1 & 0.187 & 0.195 & 0.198 \\
4 & 0.180 & 0.213 & 0.202 \\
5 & 0.215 & 0.207 & 0.200 \\
6 & 0.170 & 0.195 & 0.199 \\
7 & 0.173 & 0.195 & 0.178 \\
\hline
\end{tabular}

The reflectivity of multilayer coatings also strongly depends on the HSF roughness of the substrates. Ideally this roughness is $0.1 \mathrm{~nm}$ or less, which was the case for the substrates used in this study. On such smooth substrates multilayer coatings typically add roughness only in the HSF range, while mid- and low-spatial frequency roughness is only replicated. Atomic force microscopy (AFM) is a non-destructive technique that measures HSF roughness of the top surface, i.e. capping layer, of the multilayer coatings. Different preparations show basically identical HSF roughness $(\sim 0.18 \mathrm{~nm})$ within the measurement error of $\pm 0.02 \mathrm{~nm}$ (Table 1$)$. Roughness changes of the top layers are not directly related to reflectance, but are indicative of microstructural stability.

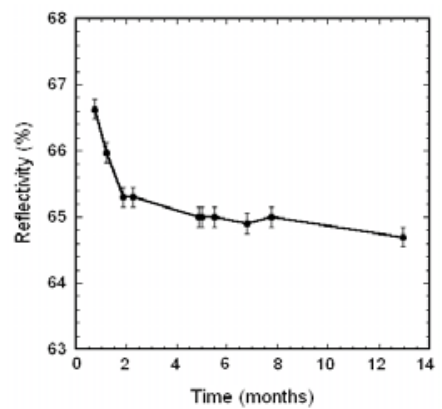

Figure 2: The aging of unexposed Ru-capped multilayer in the air at room temperature shows a drop in reflectivity of almost $2 \%$ over a period of one year.
The microstructure of Preparation 1 sample has been studied with high resolution TEM (HRTEM) in cross section and in plan view. All diffraction spots in the selected area diffraction (SAD) pattern of Preparation 1 sample obtained in cross section match with bcc Mo metal (not shown here). The strong intensity spots indicate that the crystalline, metallic Mo layers have a preferential orientation. Their $\{110\}$ crystal planes are parallel to the surface of the substrate. No diffraction rings or spots due to Ru are present. The apparent absence of a Ru signal is a sensitivity limitatin. There is only one Ru layer in 
the whole multilayer and the thickness of this layer in cross section is only $3 \mathrm{~nm}$. In the dark-field TEM imaging mode, the objective aperture is displaced so that the image is formed by a single diffracted electron beam. The dark field image thus reveals which regions of the specimen contribute to the diffracted beam, and hence, is useful for identifying crystallites of a particular orientation. An objective aperture was placed on the Mo(110) diffraction spot, which is coincident with $\mathrm{Ru}(100)$ and $\mathrm{Ru}(002)$ spots, to characterize the grain size and population of crystalline Mo and $\mathrm{Ru}$ grains. Figure 3 shows bright grains within the multilayer (Mo grains) but also in the last layer (Ru capping layer). This implies that the $\mathrm{Ru}$ layer is polycrystalline and that $\mathrm{Ru}$ grains show preferential orientation in either $\mathrm{Ru}(100), \mathrm{Ru}(002)$ or a combination of these two because these orientations have the closest match with $\mathrm{Mo}(110) \mathrm{d}$-spacing. The d-spacing for $\mathrm{Mo}(110)$ is $0.222 \mathrm{~nm}$, for $\mathrm{Ru}(100)$ is $0.23 \mathrm{~nm}$ and for $\mathrm{Ru}(002)$ is $0.214 \mathrm{~nm}$.

A cross section HRTEM image of Preparation 1 shows lattice fringes due to crystalline Ru capping layer (Figure 4). The spacing between the fringes or d-spacing on a crystal shown in Figure 4 is $\sim 0.23 \mathrm{~nm}$. This d-spacing is characteristic for $\mathrm{Ru}(100)$ crystal orientation. However, this result is not conclusive since $\mathrm{Ru}(002)$ has an almost identical d-spacing. Plan view imaging and SAD in plan view are essential to determine average grain size and to sample statistically a relevant number of grains to determine preferential grain orientation.

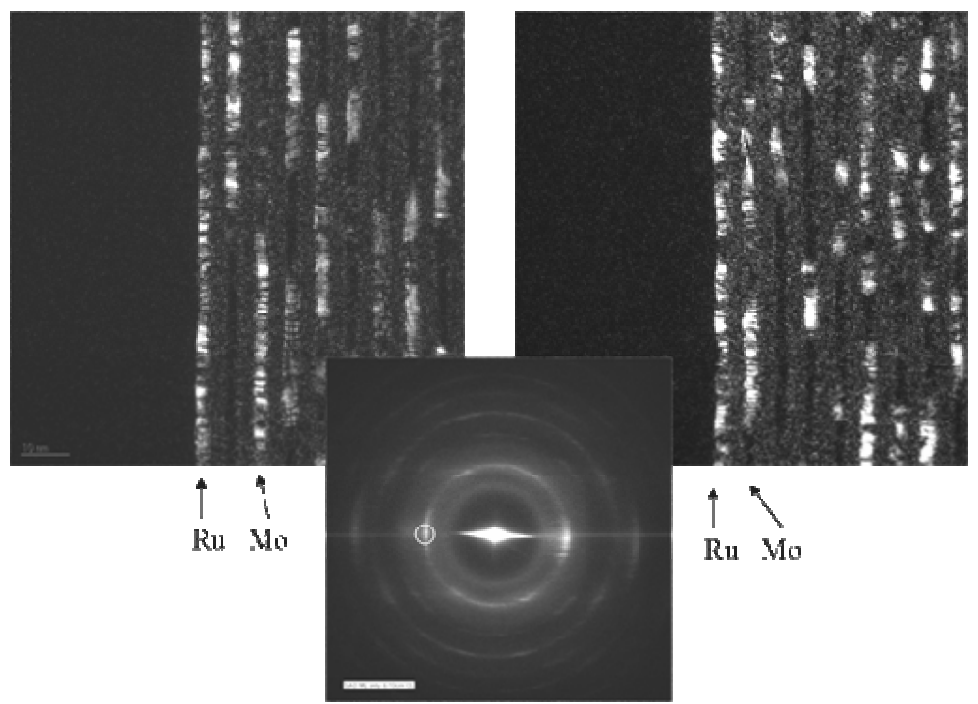

Figure 3: Two dark field images of the top few layers in Preparation 1. The diffraction pattern in the middle (bottom part) shows a circle around $\mathrm{Mo}(110)$ diffraction spot.

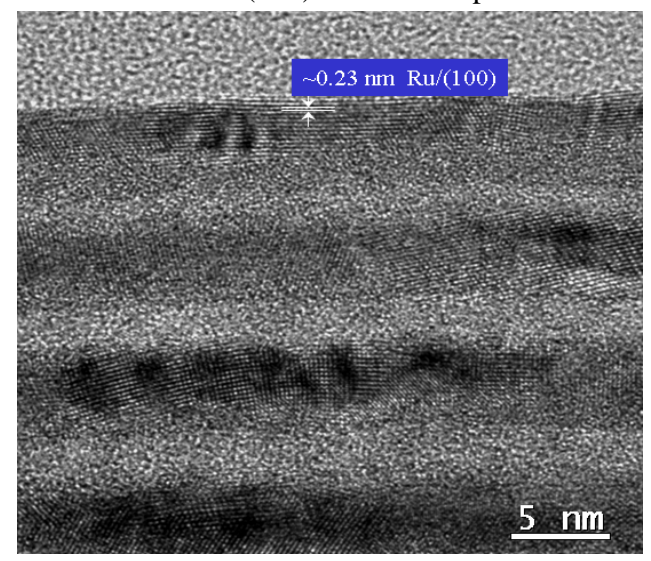

Figure 4: High resolution TEM image of the few top layers of Preparation 1 in cross section. The arrows indicate the d-spacing on a crystal in the Ru layer.
Surface chemistry was studied using PHI Quantum 2000 XPS systems at LLNL and SNL. XPS data is quantified using relative sensitivity factors and a model that assumes a homogenous layer. Photoelectrons are generated within the X-ray penetration depths (many microns), but only the photoelectrons within the top three photoelectron escape depths are detected. Hence, the analysis depths were estimated to be 5 to $10 \mathrm{~nm}$. Spectra are usually energy calibrated using the $C 1$ s signal. This could not be performed in the spectra studied here due to the interference between the $\mathrm{Ru} 3 \mathrm{~d}_{3 / 2}$ and $\mathrm{C} 1 \mathrm{~s}$ peaks. Thus the spectra were charge shifted to the $\mathrm{Si}$ $2 p$ signal, with a binding energy of $99.3 \mathrm{eV}$ for elemental Si. Initially, the survey spectra were collected from each sample in the exposed and unexposed areas. Final collection of high resolution spectra were performed only for the elements found in the survey spectra. We used the $\mathrm{O}$ 1s and $\mathrm{Ru} 3 \mathrm{p}$ peaks to obtain chemical state information especially about the oxides. No H, He or Ar was detected in any of the samples. The two major detected elements were Ru and O. Low levels of N, Si and Mo were present as well. The presence and concentration of $\mathrm{C}$ on the sample was difficult to determine due to severe interference with the $\mathrm{Ru} 3 \mathrm{~d}_{3 / 2}$ 

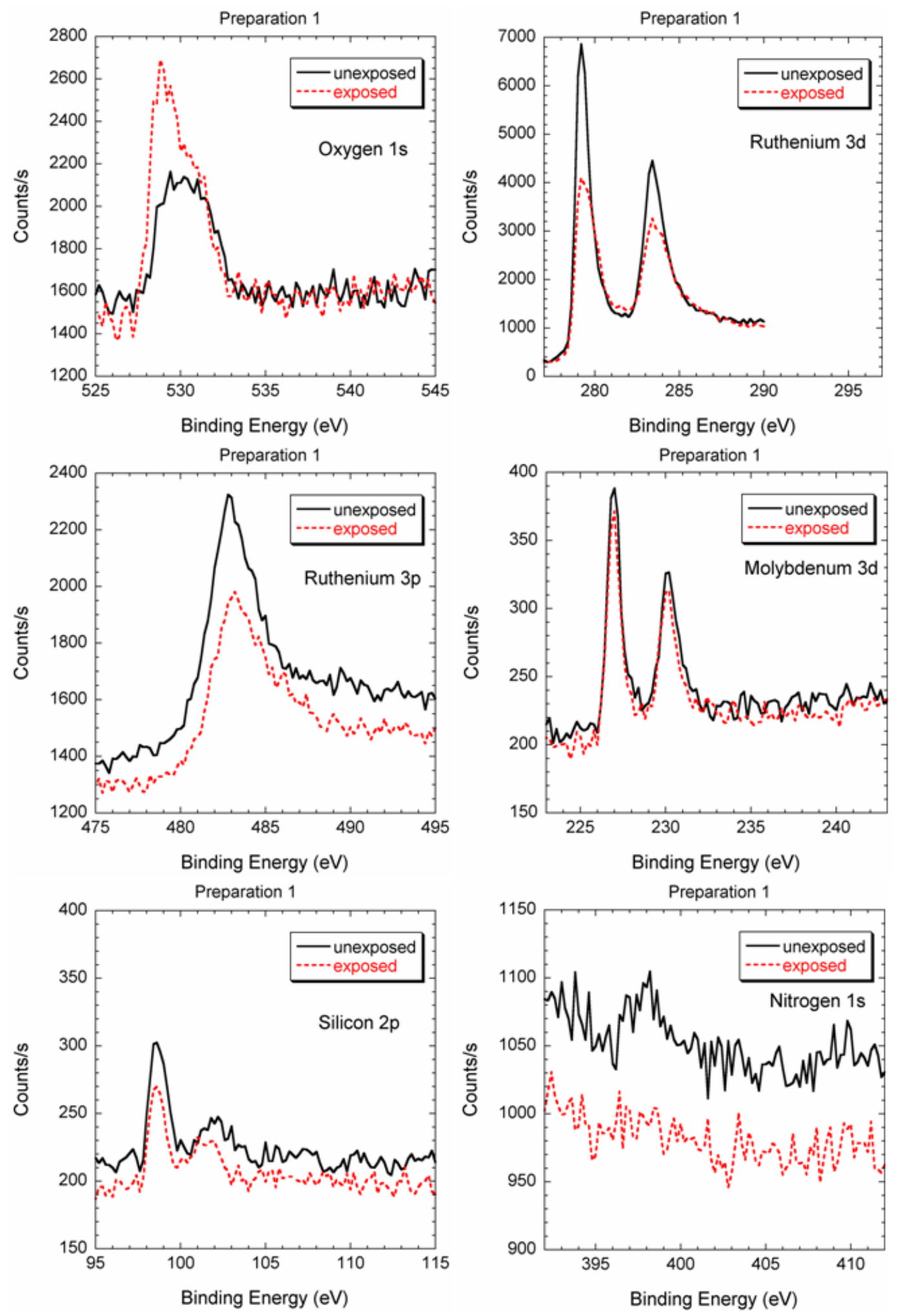

Figure 5: XPS data of O 1s, Ru 3d, Ru 3 p, Mo 3 d, Si 2 p and N 1s on Preparation 1 sample. The data in black are from asdeposited surface and the data in red are from electron beam exposed areas. 
signal. Figure 5 is an example of high resolution spectra of $\mathrm{O} 1 \mathrm{~s}, \mathrm{Ru} 3 \mathrm{~d}, \mathrm{Ru} 3 \mathrm{p}_{1 / 2}$, Mo 3d, Si 2p and N 1s XPS peaks for Preparation 1 in the electron-beam exposed and unexposed areas. With the exception of $\mathrm{N}$ all other elements show well resolved peaks.

\subsection{Oxidation Resistance Testing}

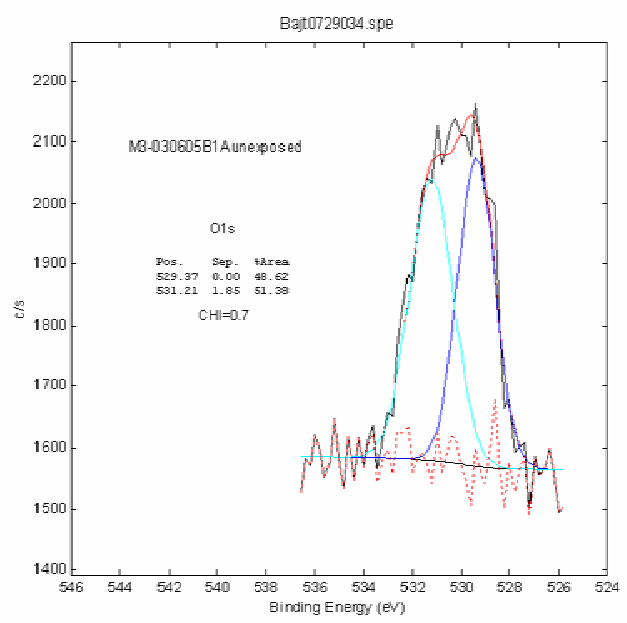

Figure 6a: High resolution XPS data on O 1s peak in unexposed, as-deposited area in Preparation 1 sample.

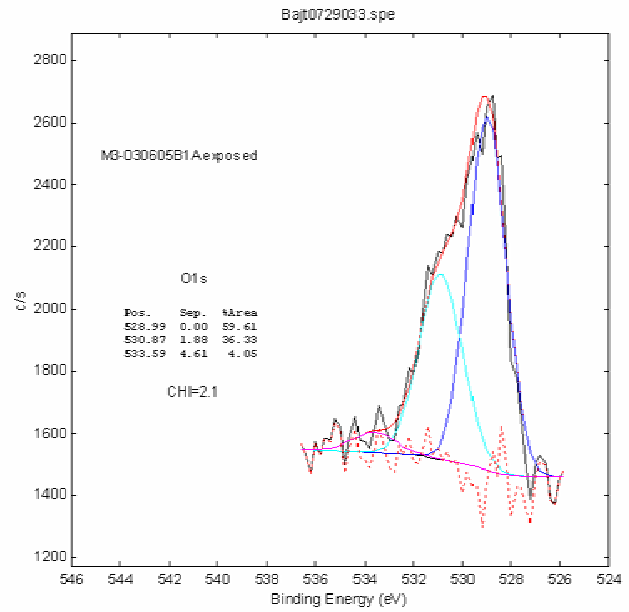

Figure 6b: High resolution XPS data on O 1s peak in exposed area in Preparation 1 sample. The fitting required three non-linear least square peaks.

The $\mathrm{Ru} 3 \mathrm{p}_{1 / 2}$ peak does not suffer from interference with other elemental peaks (unlike $\mathrm{Ru} 3 \mathrm{~d}_{3 / 2}$, which interferes with $\mathrm{C} 1 \mathrm{~s}$ ). The fits to the exposed and unexposed area spectra comparable damage to multilayers for accelerated EUV light exposures done in the same environment[6-7]. For the purpose of screening the capping layer preparations, we decided to test the multilayers under only one environmental condition using an electron beam as the source of energy. The exposures were done in an Auger microprobe chamber using $1 \mathrm{keV}$ electrons, $5 \mu \mathrm{A} / \mathrm{mm}^{2}$ current density and $5 \times 10^{-7}$ Torr water vapor. The total exposure time on each sample was 40 hours.

For Preparation 1, the major changes occur in energy position, peak separation and peak shape of $\mathrm{O} 1 \mathrm{~s}, \mathrm{Ru} 3 \mathrm{~d}$ and $\mathrm{Ru} 3 \mathrm{p}$ peaks. The $\mathrm{O} 1 \mathrm{~s}$ peak in the unexposed area could be fitted with two non-linear least square curves, one centered at $529.4 \mathrm{eV}$ and the other at $531.2 \mathrm{eV}$ (Figure 6a). The energies of these peaks are characteristic of the oxides $\mathrm{RuO}_{2}$ and $\mathrm{RuO}_{3}$, respectively. Both peaks have similar areas. In the exposed area, the fitting of $\mathrm{O} 1 \mathrm{~s}$ peak required three non-linear least square peaks centered at $529.0 \mathrm{eV}$, $530.9 \mathrm{eV}$ and $533.6 \mathrm{eV}$ (Figure 6b). The peak at $529.0 \mathrm{eV}\left(\mathrm{RuO}_{2}\right)$ is almost twice as large as the one at $531 \mathrm{eV}\left(\mathrm{RuO}_{3}\right)$, indicating preferred formation of $\mathrm{RuO}_{2}$ over $\mathrm{RuO}_{3}$ during oxidation with water. The $\mathrm{O}$ 1s peak at $533.6 \mathrm{eV}$ in the exposed area is due to $\mathrm{Si}$ oxide $\left(\mathrm{SiO}_{2-\mathrm{x}}\right)$. The non-linear square fits of $\mathrm{O} 1 \mathrm{~s}$ peak for Preparations 4, 5 , 6, and 7 (not shown) in both the unexposed and exposed areas required three instead of two Gaussian shaped peaks, indicating the presence of $\mathrm{Ru}$ and $\mathrm{Si}$ oxides in both areas for all of these preparations. The energies of these three peaks are approximately the same but their ratios for each fit are different.

The corresponding Si 2p peak for Preparation 1 has a large elemental peak at $99.3 \mathrm{eV}$, and a smaller, wider peak near $102 \mathrm{eV}$, which again corresponds to a small amount of $\mathrm{SiO}_{2-\mathrm{x}}$ (Figure 5). The $\mathrm{Si}$ oxide peak appears in the unexposed area of all multilayer preparation samples, but it is considerably smaller than the elemental Si peak for preparations 1, 6, and 7. For preparations 4 and 5, the Si $2 p$ peaks corresponding to elemental $\mathrm{Si}$ and $\mathrm{Si}$ oxide have nearly equal areas, indicating that the capping layers formed with the Ar: $\mathrm{N}_{2}$ mixture preparations have reduced oxidation resistance. Since the samples were aged in air for at least 2 months before reflectivity and XPS measurements, it is expected that there was some diffusion of $\mathrm{O}$ and $\mathrm{Si}$ into the capping layer during exposure to the air, and although not measured with XPS, the Si in the as-deposited state was probably much less oxidized than the Si after aging.

Electron beam exposures have been successfully used in the past to study lifetimes of multilayer coatings for EUVL. We have demonstrated that accelerated electron beam exposures produce

other elemental peaks (unlike Ru $3 \mathrm{~d}_{3 / 2}$, which interferes with $\mathrm{C}$ s). The fits to the exposed and unexposed area spectra 
each require two peaks, one near $483.5 \mathrm{eV}$, which includes both $\mathrm{Ru}$ and $\mathrm{RuO}_{2}$, and one near $485.9 \mathrm{eV}$ for $\mathrm{RuO}_{3}$. The size of the $\mathrm{Ru}$ oxide component relative to the elemental $\mathrm{Ru}$ component is increased for the exposed area spectrum. This indicates further oxidation of the Ru capping layer with exposure. In addition, although the Ru $3 d$ peaks are not fit, the significant reduction of their intensity in the exposed area relative to the unexposed area indicates an increased coverage of oxide. This is because an oxide layer would reduce the intensity of underlying layers due to the limited escape depth of the photoelectrons $(5-10 \mathrm{~nm})$.

The Si $2 p$ peak is very similar in the exposed and unexposed areas for Preparation 1, indicating very little further oxidation of the $\mathrm{Si}$. The $\mathrm{O} 1 \mathrm{~s}$ and $\mathrm{Ru} 3 \mathrm{p}_{1 / 2}$ results also are consistent with oxidation of the Ru but not the Si in the exposed area. Preparation 6 had similar O 1s, Ru 3p $p_{1 / 2}$, and Si 2p spectra to Preparation 1, indicating good oxidation resistance of the Preparation 6 capping layer and additional $\mathrm{RuO}_{2}$ formation as well. Preparation 7 had a large increase of the Si oxide component of the Si 2p peak, and preparations 4 and 5 had smaller increases, indicating that the oxidation resistance of capping layers formed using these three preparations is reduced relative to those formed using Preparations 1 and 6. While Preparation 7 (Ru capping layer with carbon doping) was more resistant to oxidation from aging in the air, it was much less resistant to oxidation from electron beam plus water exposure.

For all preparations, the Mo 3d peak is unchanged in the unexposed and exposed areas, and is characteristic of metallic Mo with no Mo oxide formed. This indicates that for all preparations the oxidation does not extend as far as the first Mo layer, which is beneath both the capping layer and the first Si layer (Figure 5).

$\mathrm{N}$ 1s spectra have also been obtained for all capping layer preparations. The $\mathrm{N}$ 1s spectra for Preparations 1 and 7 are quite weak and are due to residual $\mathrm{N}_{2}$ in the deposition chamber, since $\mathrm{N}_{2}$ was not deliberately used in these preparations. Preparations 4 and 5 exhibit a rather strong $\mathrm{N}$ 1s peak in both the unexposed and exposed area. This is expected since the capping layers for Preparation 4 and 5 were deposited in an Ar: $\mathrm{N}_{2}$ environment, so some incorporation of $\mathrm{N}$ could occur. For preparation 6 , the $\mathrm{N} 1$ s peak is strong in the unexposed area but disappears in the exposed area. This suggests that nitrogen was weakly absorbed on capping layer surface during the post-deposition $\mathrm{N}_{2}$ exposure of the Preparation 6 surface in the deposition chamber.
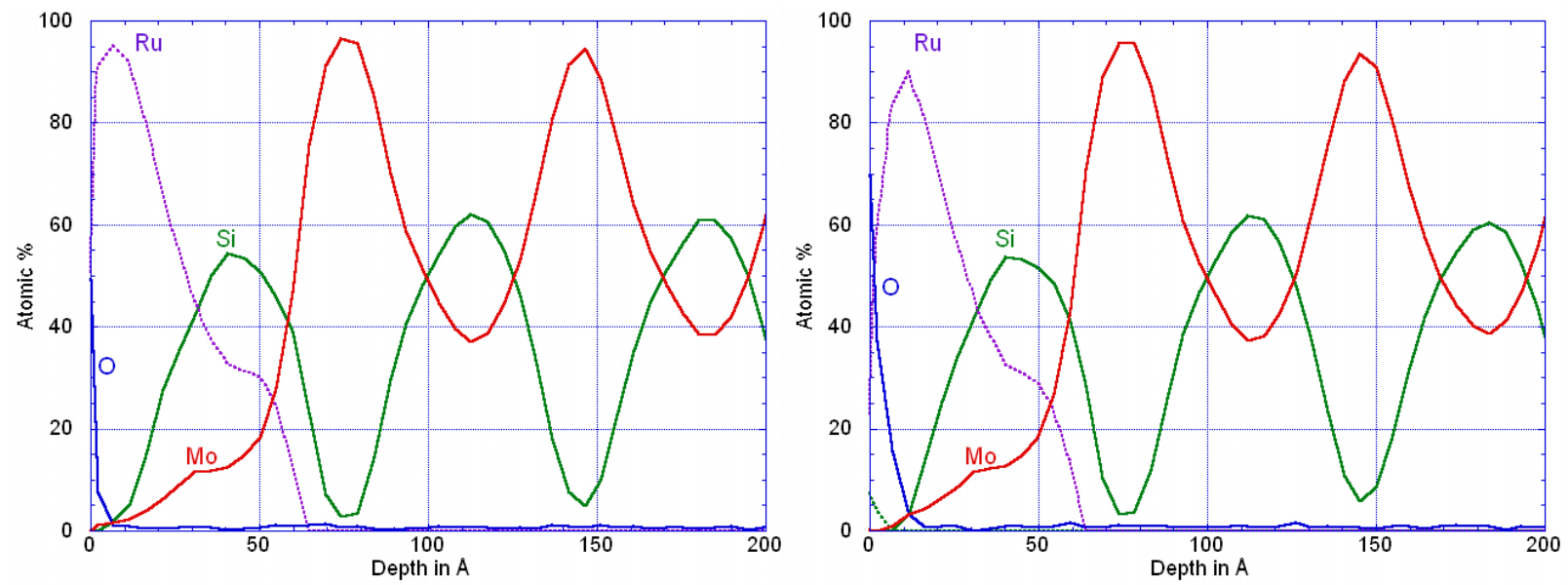

Figure 7: Auger depth profile on Preparation 1 sample in as-deposited, unexposed area (left plot) and in an electron beam exposed area (right plot).

Lastly, Auger depth profiling was performed to determine interface sharpness and thicknesses of the oxide, the capping layer, and the multilayer. Auger depth profiling was performed only on Preparations 1 and 6 . Figure 7 shows the results of Preparation 1 in the unexposed and exposed areas, respectively. The Auger depth profiles in the unexposed and exposed areas are strikingly similar. The underlying well-defined Mo/Si multilayer (depth greater than $7.0 \mathrm{~nm}$ ) is unaffected by the electron beam exposure, as is the shape of the Ru, Mo, and Si profile in the layer between $1.5 \mathrm{~nm}$ and $7.0 \mathrm{~nm}$. Oxygen is observed only in the top 1.0-1.5 nm of Ru of the exposed area, and only in the top 0.5 $\mathrm{nm}$ of the unexposed area. There is some diffusion of Si and Mo into the Ru capping layer as well, which may account for the observation of oxidized Si in the unexposed and exposed areas of O 1s and Si 2p XPS data. The underlying Si in 
the multilayer clearly is not oxidized. Preparation 6 has a very similar depth profile to Preparation 1 in terms of the depth of the oxygen into the Ru capping layer and the lack of disturbance of the underlying multilayer.

\subsection{Thermal Testing}

Thermal tests were performed to study the effect of temperature on different preparations. The wavefront of the projection optics is extremely sensitive to any change in the temperature. The temperature in the projection optics box will likely be maintained at room temperature within a fraction of a degree. However, knowledge about the thermal stability of capping layers is of interest because optics could be potentially cured at higher temperature to relieve stress or stabilize the multilayer performance [11]. Samples with different capping layer preparations were annealed at $100^{\circ} \mathrm{C}$ for 1 week and $200^{\circ} \mathrm{C}$ for 30 minutes. The annealing temperatures were controlled within $\pm 5^{\circ} \mathrm{C}$ and all experiments were performed in relatively good vacuum (base pressure $\sim 3 \times 10^{-8}$ Torr). We monitored changes in period thickness, interface roughness, inter-diffusion, EUV reflectance and surface roughness.

Table 2: Reflectance loss (absolute percent) for Preparations 1-6 shows differences due to differences in capping layer structures.

\begin{tabular}{|ccc|}
\hline Preparation & $\Delta \mathrm{R}(\%)$ for $100^{\circ} \mathrm{C}$ & $\Delta \mathrm{R}(\%)$ for $200^{\circ} \mathrm{C}$ \\
1 & -1.00 & -1.60 \\
4 & -0.80 & -1.68 \\
5 & -1.05 & -2.04 \\
6 & -1.07 & -1.74 \\
7 & -2.10 & -3.54 \\
\hline
\end{tabular}

Figure 8 shows absolute wavelengths for as-deposited samples, samples annealed at $100^{\circ} \mathrm{C}$ for 1 week and samples annealed at $200^{\circ} \mathrm{C}$ for 30 minutes for different preparation techniques. The wavelength shifts are similar for all five samples. The wavelength, which was about $13.4 \mathrm{~nm}$ in as-deposited samples shifted to $\sim 13.35 \mathrm{~nm}$ after $100^{\circ} \mathrm{C}$ for 1 week. The wavelength of the multilayers exposed to $200^{\circ} \mathrm{C}$ for 30 minutes changed from $13.4 \mathrm{~nm}$ to $\sim 13.28 \mathrm{~nm}$. The data clearly show that the peak wavelength shifted by the same amount on all samples. This attests that the multilayer structures, excluding the capping layer, were the same and that the annealing conditions were very reproducible. As expected, samples annealed to $200^{\circ} \mathrm{C}$ for 30 minutes show a larger shift in the peak wavelength than samples annealed at $100^{\circ} \mathrm{C}$. Annealing at $100^{\circ} \mathrm{C}$ causes about $1 \%$ drop in reflectivity in all samples except in Preparation 7 sample, where the reflectivity loss is about twice as much (Table 2). Testing at higher temperature $\left(200^{\circ} \mathrm{C}\right)$ shows good thermal stability of Preparations 1, 4 and 6 with about $\sim 1.7 \%$ reflectance loss. Preparation 5 shows reflectance loss of $2 \%$ and Preparation 7 about 3.5\% reflectance loss. This suggests that differences in reflectivity are due to different capping layer preparations and that higher temperatures accelerate the mechanism that leads to reduced reflectivity. The HSF roughness is slightly increased for annealed samples as compared to as-deposited samples. However, the increase is still within the measurement error of $\pm 0.02 \mathrm{~nm}$ (Table 1). Hence, reflectance loss due to annealing can not be explained

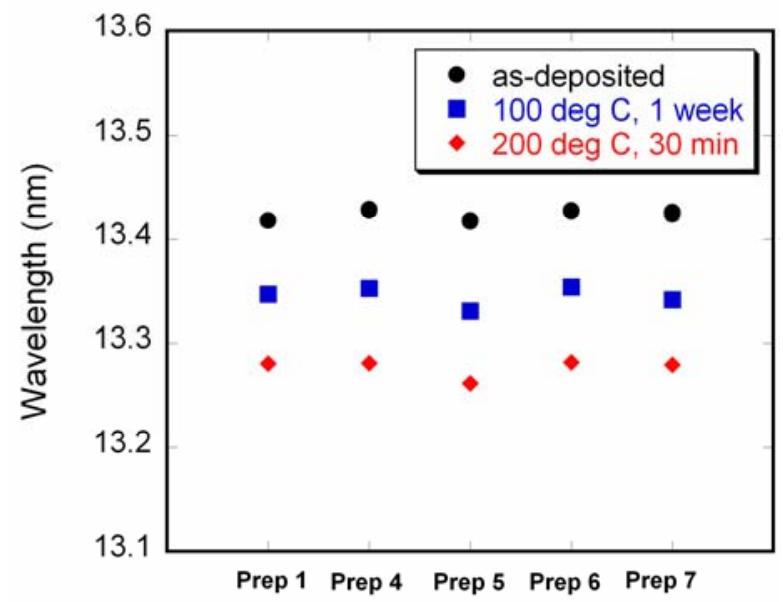

Figure 8: Absolute wavelengths for as-deposited and annealed samples with different capping layer preparations. with increase in surface roughness.

\section{CONCLUSIONS}

The lifetime of projection optics remains high on a list of unresolved critical issues for EUVL technology. Development of oxidation resistant capping layers requires understanding of the microstructure of the capping layers and the oxidation mechanisms. We studied the effect of different preparations of Ru capping layers on their thermal and oxidation resistance and report the preliminary results. The highest reflectivity was measured on Preparation 7 
(ruthenium doped with carbon), which was very stable against aging in the air. However, this capping layer preparation had disappointing performance when exposed to the electron beam in water vapor and when exposed to high temperature. In the electron beam exposed area, an increase in Si oxide was observed. Since Si layer is below the capping layer, this might suggest that the diffusion of oxygen through this capping layer is faster than in other preparations studied here. Ru capping layers deposited with Ar: $\mathrm{N}_{2}$ sputtering gas mixture (Preparation 4 and 5) had low initial reflectivity. TEM images in these capping layers show smaller, randomly oriented Ru grains. Silicon underneath the Ru capping layer is partly oxidized even in as-deposited state. In the electron beam exposed areas we observe an increase in Si oxide underneath the Ru capping layer.

Preparation 1 and 6 samples show identical initial reflectivity. Preparation 6 sample was exposed to $\mathrm{N}_{2}$ gas before taken out of the deposition chamber. Although we could see some $\mathrm{N}$ bonded to the surface of as-deposited sample this difference in surface chemistry as compared to Preparation 1 did not affect the performance of the Preparation 6 capping layer when exposed to the electron beam in the presence of water vapor. Preparation 1 had overall the best performance for thermal and oxidation stability and was therefore studied in most detail. The microstructure of the as-deposited sample was studied in cross section using HRTEM and with SAD in plan view. Preliminary results suggest that Ru in Preparation 1 is crystalline and has preferential growth orientation, either Ru (100) or Ru (002). The electron beam exposed area showed no increase in Si oxide underneath the Ru capping layer, unlike what we observed in Preparations 4, 5, and 7. Based on limited TEM data of other preparations, we conclude that the microstructure plays a crucial role in oxidation resistance of the capping layers. It appears that a capping layer with randomly oriented grains is less oxidation resistant than a capping layer with well developed texture, such as Preparation 1.

\section{ACKNOWLEDGMENTS}

We would like to thank Cindy Larson, Cheryl Evans, Regina Soufli, Art Nelson, Rick Gross (all LLNL) and Patrick A. Kearney (now at SEMATECH) for their contributions. We also appreciate discussions and support from Stefan Wurm and Obert Wood (SEMATECH). This work was performed under the auspices of the U.S. Department of Energy by University of California Lawrence Livermore National Laboratory under contract No. W-7405-Eng-48. This project was funded by SEMATECH under Project LITH 160.

\section{REFERENCES}

1. S. Graham, C. Steinhaus, M. Clift, and L. Klebanoff, "Radio-frquency discharge cleaning of silicon-capped Mo/Si multilayer extreme ultraviolet optics”, J. Vac. Sci. Technol., B20, 2393-2400 (2002).

2. B. M. Mertens, B. van der Zwan, P. W. H. de Jager, M. Leenders, H. G. C. Werij, J. P. H. Benschop, and A. J. J. van Dijsseldonk, "Mitigation of surface contamination from resists outgassing in EUV lithography", Microelectron. Eng. 53, 659-662 (2000).

3. N. Koster, B. Mertens, R. Jansen, A. van de Runstraat, F. Stietz, M. Wedowski, H. Meiling, R. Klein, A. Gottwald, F. Scholze, M. Visser, R. Kurt, P. Zalm, E. Louis, A. Yakshin, "Molecular contaminaton mitigation in EUVL by environmental control”, Microelectron. Eng. 61-61, 65-76 (2002).

4. L. E. Klebanoff, W. M. Clift, M. E. Malinowski, C. Steinhaus, S. Bajt, "Radiation-induced protective carbon coating for extreme ultraviolet optics”, J. Vac. Sci. Technol. B20, 695-703 (2002).

5. L. E. Klebanoff, M. E. Malinowski, W. M. Clift, C. Steinhaus, and P. Grunow, "Use of gas-phase ethanol to mitigate extreme UV/water oxidations of extreme UV optics”, J. Vac. Sci. technol. A22, 425-432 (2004).

6. S. Bajt, H. N. Chapman, N. Nguyen, J. Alameda, J. C. Robinson, M. Malinowski, E. Gullikson, A. Aquila, C. Tarrio, S. Grantham, "Design and Performance of Capping Layers for EUV Multilayer Mirrors," in Emerging Lithographic Technologies VII, R. L. Engelstad, ed., Proc. SPIE 5037, 236-248 (2003).

7. S. Bajt, H. N. Chapman, N. Nguyen, J. Alameda, J. C. Robinson, M. Malinowski, E. Gullikson, A. Aquila, C. Tarrio, and S. Grantham, Applied Optics 42, (2003). 
8. B. M. Mertens, N. Koster, R. Jansen, A. van de Runstraat, H. Werij, F. Stietz, M. Wedowski, H. Meiling, R. Klein, R. Thornagel, F. Scholze, G. Ulm, R. Kurt, P. Zalm, E. Louis, A. Yakshiun, in : ASET/SEMATECH Workshop on EUV Lithography, Matsue, 2001, O4-5.

9. A. E. Yakshin, E. Louis, E. L. G. Maas, F. Bijkerk, R. Klein, F. Scholze, P. Zalm, F. Stietz, M. Wedowski, S. Muellender, B. Mertens, H. Meiling, in ASET/SEMATECH Workshop on EUV Lithography, Matsue, 2001, P6-6.

10. P. B. Mirkarimi, "Stress, reflectance, and temporal stability of sputter-deposited Mo/Si and Mo/Be multilayer films for extreme ultraviolet lithography”, Opt. Eng. 38, 1246-1259 (1999).

11. S. Bajt et al., in preparation. 